REVISTA

MEXICANA DE

ECONOMÍA Y

FINANZAS

REMEF

(THE MIEXICAN JOURNAL OF

ECONOMICS AND FINANCE)
Revista Mexicana de Economía y Finanzas, Nueva Época

Volumen 16 Número 4, Octubre - Diciembre 2021, pp. 1-16, e656

DOI: https://doi.org/10.21919/remef.v16i4.656

(Recibido: 9/noviembre/2020, aceptado: 1/marzo/2021, publicado: 10/mayo/2021)

\title{
Decisiones fiscales, crecimiento económico y desigualdad del ingreso
}

\author{
Juan Marroquín-Arreola ${ }^{1}$ - Instituto Politécnico Nacional, México \\ Humberto Ríos Bolívar² - Instituto Politécnico Nacional, México
}

El objetivo de este artículo es analizar de qué manera los instrumentos de política fiscal influyen en el crecimiento y en la desigualdad del ingreso en México. Para lo cual se presentan los antecedentes teóricos y se utiliza un modelo de crecimiento endógeno que incorpora variables fiscales. Asimismo, se estima un modelo econométrico mediante la técnica de datos de panel. Los resultados empíricos muestran que; el gasto corriente no impacta al crecimiento económico; los impuestos directos ejercen un impacto negativo en el crecimiento, mientras que los impuestos indirectos no arrojan resultados significativos en el modelo; el gasto corriente contribuye a reducir la desigualdad; los impuestos directos contribuyen a una reducción de la desigualdad, mientras que los impuestos indirectos contribuyen a aumentarla. La originalidad y principal contribución de este trabajo radica en su enfoque por entidad federativa considerando aspectos redistributivos. La limitante de este tipo de estudios se debe a la escasez los datos fiscales en algunas entidades federativas. Se recomienda considerar otras variables para determinar el causal entre eficiencia y equidad cuando se determinan variables fiscales.

Clasificación JEL: E62, D63, 047.

Palabras clave: política fiscal, crecimiento económico, desigualdad.

\section{Fiscal Decisions, Economic Growth and Income Inequality}

The aim of this paper is to analyze how fiscal policy instruments influence growth and income inequality in Mexico. For which the theoretical background is presented and an endogenous growth model that incorporates fiscal variables is used. Likewise, an econometric model is estimated using the panel data technique. The empirical results show that; current spending does not impact economic growth; direct taxes have a negative impact on growth, while indirect taxes do not show significant results in the model; current spending contributes to reducing inequality; direct taxes contribute to a reduction in inequality, while indirect taxes contribute to increasing it. The originality and main contribution of this document lies in its approach by federal entity considering redistributive aspects. The limitation of this type of study is due to the scarcity of fiscal data in some states. It is recommended to consider other variables to determine the causal between efficiency and equity when determining fiscal variables.

JEL Classification: E62, D63, 047.

Keywords: fiscal policy, economic growth, inequality.

\footnotetext{
${ }^{1}$ Autor de correspondencia. Profesor-investigador en la Escuela Superior de Economía del Instituto Politécnico Nacional. Correo: juanmarro@gmail.com

2 Profesor-investigador en la Escuela Superior de Economía del Instituto Politécnico Nacional. Correo: hrios@ipn.mx

*Sin Fuente de financiamiento para el desarrollo de la investigación
} 


\section{Introducción}

En la literatura económica existen diferentes enfoques teóricos donde se aborda la interrelación entre la política fiscal, la desigualdad del ingreso y el crecimiento económico. Uno de esos enfoques es la hipótesis de equivalencia ricardiana, la cual menciona que la política fiscal no tiene efectos positivos significativos en la demanda agregada y, por lo tanto, tampoco lo tiene en el crecimiento económico. Esto debido a que hay efectos neutrales de las estrategias de financiamiento de deuda o impuestos para estimular la demanda agregada ya que los consumidores tienden a internalizar la estrategia fiscal del gobierno al tomar sus decisiones de ahorro para futuros pagos de impuestos. Otro enfoque es el modelo Keynesiano, el cual afirma que la inversión del gobierno y el consumo que se financian con deudas estimulan la demanda agregada, lo cual a su vez promueve el crecimiento económico. Por su parte, la hipótesis de Kuznets (1955) postula que el crecimiento económico conduce a un aumento de la desigualdad de ingresos en las primeras etapas del desarrollo y tiende a disminuir dicha desigualdad en las últimas etapas del desarrollo.

Por otro lado, desde un enfoque neoclásico, algunos modelos destacan los efectos a corto plazo de distintos instrumentos de política fiscal. En este enfoque, el crecimiento en estado estacionario es inducido por factores exógenos como los cambios en el crecimiento de la población y el progreso tecnológico. Entre estos modelos destacan, por ejemplo; Sato (1967) y Feldstein (1974) que utilizan el modelo neoclásico para analizar los efectos de diferentes impuestos sobre el crecimiento económico. Por su parte, Summers (1981) y, Auerbach y Kotlikoff (1987) adecuan el modelo de generaciones traslapadas de Diamond (1965) para analizar los efectos dinámicos de la política fiscal en el crecimiento. Asimismo, Chamely (1986), Judd (1985) utilizaron el modelo de Cass y Koopmans (1965) para estudiar los efectos de la política fiscal considerando tasas de ahorro endógeno.

Por otra parte, están los modelos de crecimiento endógenos, que difieren de los modelos de crecimiento neoclásico de política pública debido que en los endógenos el crecimiento no se realiza por factores exógenos. En los modelos de crecimiento endógeno la inversión en capital humano y físico afecta la tasa de crecimiento en estado estacionario y, en consecuencia, hay mucho más margen para que los gastos fiscales y gubernamentales desempeñen un papel determinante en el proceso de crecimiento. Esto tiende a transformar los efectos de crecimiento temporal de la política fiscal que involucra el modelo neoclásico, en efectos permanentes. Por lo tanto, los modelos de crecimiento endógeno que incorporan políticas públicas predicen que distorsionar los impuestos, así como los gastos públicos productivos, afectan el crecimiento económico. De esto se deduce que la política fiscal puede afectar el nivel de producción, así como su tasa de crecimiento a largo plazo. Desde las contribuciones pioneras de Barro (1990), King y Rebelo (1990) y Lucas (1990), varios documentos han extendido el análisis de los impuestos, el gasto público y el crecimiento (García-Peñalosa y Turnovsky, 2007). Por último, Venegas-Martínez (2010), (2009) y (2005) examina el impacto de la política fiscal en el crecimiento en un modelo estocástico de crecimiento endógeno.

La política fiscal afecta la desigualdad tanto directa como indirectamente. Los instrumentos de política fiscal (impuestos, subsidios, transferencias y gastos) tienen roles redistributivos directos. Como lo destaca el Fondo Monetario Internacional (FMI), "La política fiscal puede ayudar a mejorar la redistribución al reducir las desigualdades de ingresos disponibles (después de impuestos y 
transferencias) y de mercado (antes de impuestos y transferencias). Los impuestos y las transferencias relacionadas con los ingresos afectan la desigualdad del ingreso disponible, mientras que las transferencias en especie, como el gasto en salud y educación, influyen en la desigualdad de los ingresos del mercado. La política fiscal puede ser un poderoso instrumento redistributivo"3.

Diversas investigaciones teóricas que estudian la relación entre desigualdad y crecimiento económico tienen resultados opuestos. Por ejemplo, algunos estudios señalan que una mayor desigualdad inicial fomenta el crecimiento económico (Rebelo, 1991; Deaton y Paxson, 1997), otros mencionan que una desigualdad inicial podría ser perjudicial para el crecimiento (Aghion y Bolton ,1997; Piketty,1997; Galor y Zang,1997; Mookherjee y Ray, 2003). Por parte de la evidencia empírica hay estudios que utilizan modelos econométricos que muestran que en el largo plazo hay un impacto negativo de la desigualdad en el crecimiento económico (Persson y Tabellini,1994; Perotti, 1996). Asimismo, otros estudios muestran resultados distintos donde el crecimiento contribuye a reducir la desigualdad (Lin et al.,2009; Huang et al., 2009; Castelló-Climent,2010).

La desigualdad es un tema polémico que produce gran interés en muchas partes del mundo. Este interés se produce a raíz de los considerables aumentos en las disparidades de ingresos que se ha observado durante las últimas tres décadas. Los formuladores de políticas enfrentan desafíos difíciles para comprender cómo lidiar con la desigualdad, como mejorar la distribución del ingreso y como lograr altas tasas de crecimiento económico y finanzas públicas sostenibles.

El objetivo de este artículo es analizar de qué manera instrumentos de política fiscal influyen crecimiento y en la desigualdad del ingreso en México. Par lo cual se exponen antecedentes teóricos y se utiliza un modelo de crecimiento endógeno que incorpora variables fiscales. Asimismo, se información de 32 entidades y aplicación de técnicas econométricas para evaluar la incidencia de variables fiscales en crecimiento y desigualdad.

El documento se estructura de la siguiente manera. En la segunda sección, ser presenta un modelo teórico de crecimiento endógeno que incorpora variables fiscales. En la tercera sección, se muestra la evolución y comportamiento de la desigualdad y el crecimiento. En cuarta sección, se realiza un análisis econométrico mediante un panel de datos. Finalmente, se exponen las conclusiones.

\section{Breve revisión de literatura}

En esta sección se expondrá una breve revisión de estudios que versan sobre la relación entre la política fiscal, el crecimiento y la desigualdad de ingreso. En la literatura se encuentran una gran variedad estudios que analizan los efectos empíricos de las políticas fiscales, pero con resultados contrapuestos. Easterly y Rebelo (1993) analizan el efecto de las políticas fiscales en distintos países, agregan variables fiscales de manera ad-hoc a una ecuación empírica de crecimiento, los resultados muestran que el impacto y la significancia de las variables fiscales dependen del conjunto de variables de control incluidas y de las condiciones iniciales en que se encuentre la economía. Chu et al. (2000), utilizando una muestra de economías en desarrollo, encuentran un impacto significativo de los impuestos directos en la reducción de la desigualdad, pero estos efectos también son de magnitud extremadamente pequeña. De igual manera, Li et al. (2000) utilizan un panel de 84 países que incluye

\footnotetext{
${ }^{3}$ IMF (2017), Fiscal Monitor (October), p. 1
} 
países desarrollados y en desarrollo y no obtienen efectos estadísticamente significativos del gasto público total en la distribución del ingreso. Por su parte, Chung y Turnovsky (2010) analizaron los efectos de las políticas de crecimiento utilizando un modelo de crecimiento endógeno de equilibrio general. Sus resultados sugieren que el gasto público en capital público aumentará la desigualdad de la riqueza gradualmente, independientemente de cómo se financie. La inversión gubernamental tiende a mejorar la productividad del capital privado, estimulando así su acumulación, y el capital privado se distribuye de manera más desigual entre los agentes que el trabajo, por lo que tiende a aumentar la desigualdad de la riqueza.

Wolff y Zacharias (2007) evalúan los efectos de los gastos del gobierno de los Estados Unidos y los impuestos sobre los hogares entre los años 1989 y 2000. Ellos utilizan modelos econométricos para hacer una estimación de gastos netos del gobierno conforme a los ingresos ajustados de acuerdo con la riqueza, los resultados muestran que la desigualdad general de ingresos disminuyó significativamente en consonancia con el gasto público neto. El análisis de los desgloses sugirió que los gastos, en comparación con los impuestos, producen mayores resultados de reducción de la desigualdad.

Barreix et al. (2007) analizan el caso de los países andinos (Colombia, Venezuela, Ecuador, Perú, Bolivia, Argentina, Chile), y concluyen que el efecto total de los impuestos es levemente regresivo, debido a una débil capacidad de recaudación del impuesto sobre la renta. Asimismo, Goñi et al. (2008) analizan a países de América Latina tales como México, Colombia, Chile, Argentina, Perú y Brasil, Chile, en sus resultados sugieren que el bajo desempeño de las políticas redistributivas de debe al poco volumen de recursos recaudados y transferidos, la existencia de impuestos regresivos y transferencias mal orientadas. Cubero y Hollar (2010) estudian el caso de los países centroamericanos, ellos encuentran que encuentran que el impacto del gasto social en la pobreza y la distribución del ingreso se ve socavado por su nivel absoluto relativamente bajo.

Alesina y Ardagna (2009) examinaron casos de estímulos y ajustes fiscales en países de la OCDE entre 1970 y 2007. Ellos concluyen que los ajustes fiscales basados en recortes de gastos acompañados de recortes de impuestos son la forma más exitosa de adaptación de políticas.

Lopéz et al. (2010), analizan en caso de 40 países en desarrollo, muestran que la reasignación del gasto público de bienes privados y subsidios no sociales a bienes públicos y semipúblicos se asocia con reducciones de la pobreza, aunque su efecto redistributivo es neutral, lo que implica una focalización deficiente.

Kitao (2010) analizó si la política fiscal temporal, la reducción de impuestos y la transferencia de devoluciones, para estimular las actividades económicas pueden mejorar la recuperación económica. Él menciona que una reducción de los impuestos sobre la renta estipula un incentivo inmediato para trabajar y ahorrar más, lo que a su vez aumenta la producción y el consumo agregado. Concluye que ambas políticas mejoran el bienestar general de los hogares y la política de descuentos beneficia especialmente a los hogares de bajos ingresos.

En estudios realizados para el caso de México, Brosio et al. (2017) analizan el efecto de la política fiscal sobre la desigualdad personal y las disparidades territoriales, a través del impacto diferencial de la política de ingresos y gastos públicos sobre estas dos dimensiones de la desigualdad, concluye que el efecto de la política fiscal sobre la desigualdad personal y las disparidades territoriales, a través del impacto diferencial de la política de ingresos y gastos públicos sobre estas 
dos dimensiones de la desigualdad. Por su parte, Levy (2016) discute las propuestas de Keynes sobre política fiscal y analiza el impacto que dichas políticas tuvieron sobre el crecimiento económico en países en desarrollo, particularmente en México, concluye que la intervención pública en la economía debe ser reconsiderada en el ámbito del crecimiento económico. Menciona que de acuerdo con el argumento Keynesiano la política fiscal no debe estar acotada a periodos recesivos, sino que debe ser constante y debe atender los desequilibrios estructurales de la economía y canalizarse al gasto de bienes de capital, buscando construir encadenamientos con el sector productivo, sobre la base de salarios remunerativos.

\section{Desarrollo del Modelo}

En el presente apartado se expone un modelo de crecimiento endógeno de manera teórica donde se determinan un conjunto de ecuaciones que muestran la relación entre fiscales y el crecimiento y desigualdad del ingreso.

El modelo aquí desarrollado toma como referencia a Muinelo y Roca (2011). En el modelo se considera una economía de z productores, cada uno produciendo un producto $q$, de acuerdo con la siguiente función de producción:

$$
Q=T p^{1-\alpha} g^{\alpha}
$$

Donde $Q$ representa el producto, $p$ el capital privado y $g$ el capital público usado por el productor. El gobierno equilibra su presupuesto en cada período al aumentar un impuesto proporcional sobre la producción a la tasa $i$ (impuesto distorsionador) y los impuestos de suma fija $S$. Por lo tanto, la restricción presupuestaria que enfrenta el gobierno se puede expresar como:

$$
z g+d+e=S+i z q
$$

donde $d$ y $g$ son el gasto público no productivo y productivo, respectivamente. Debido a que se permite el caso de un presupuesto equilibrado o desequilibrado, se incluye una variable $e$ que recoge el superávit o déficit presupuestario.

La tasa de crecimiento del país $i$ durante el periodo $t, \Delta q_{i, t}$ es una función de un conjunto de variables no fiscales $N F_{i, t}$, y un vector de variables fiscales $V F_{i, t}$ :

$$
\Delta q_{i, t}=A+B \sum_{a=1}^{j} N F_{i, t}^{a}+\sum_{b=1}^{k} C_{b} V F_{i, t}^{b}+\varepsilon_{i, t}
$$

Luego, si se supone que el vector $V F$ incluye todos los elementos relevantes, se deduce que:

$$
\sum_{b=1}^{k} V F_{i, t}^{b}=0
$$

Para evitar una colinealidad perfecta en la estimación de la ecuación (3), se excluye un elemento del vector $V F$. La variable omitida es efectivamente el elemento compensatorio asumido 
dentro de la restricción presupuestaria del gobierno (Kneller et al., 1999). Por lo tanto, se considera que la ecuación de crecimiento se puede expresar como:

$$
\Delta q_{i, t}=A+B \sum_{a=1}^{j} N F_{i, t}^{a}+\sum_{b=1}^{k-1} C_{b} V F_{i, t}^{b}+C_{k} V F_{i, t}^{k}+\varepsilon_{i, t}
$$

Luego, si se omite el elemento $V F_{i, t}^{k}$ para obtener la nueva ecuación de crecimiento, se tiene

$$
\Delta q_{i, t}=A+B \sum_{a=1}^{j} N F_{i, t}^{a}+\sum_{b=1}^{k-1}\left(C_{b}-C_{k}\right) V F_{i, t}^{b}+\varepsilon_{i, t}
$$

Según esta estrategia, la interpretación del coeficiente estimado de cada variable fiscal es el efecto de un cambio unitario en la variable dependiente compensado por un cambio unitario en la variable fiscal omitida, que es el elemento implícito.

Para reducir el sesgo de error de especificación, en el modelo se consideran dos ecuaciones de crecimiento que contienen diferentes conjuntos de variables de control. El Modelo 1 considera un conjunto de variables de control basadas en el modelo de crecimiento de Solow que incluye el nivel inicial del PIB per cápita, la inversión privada y el crecimiento de la población. Basado en Mankiw, Romer y Weil (1992), y para controlar el impacto de la acumulación de capital humano, el Modelo 2 incluye el antiguo conjunto de Solow e incorpora los años promedio de escolaridad de la población de 25 años o más.

También en el modelo se considera la inclusión de una medida de desigualdad en las ecuaciones de crecimiento que permite un análisis conjunto de los efectos macroeconómicos y distributivos de la política fiscal. Por lo tanto, los Modelos 3 y 4 amplían ecuaciones de crecimiento con la adición de una variable de desigualdad (índice de Gini).

Para la desigualdad económica, la ecuación de referencia se basa en los enfoques empíricos Lundberg y Squire (2003). Las variables de política fiscal se incorporan siguiendo la misma estrategia utilizada para la ecuación de crecimiento. Por lo tanto, el rendimiento de la desigualdad de ingresos depende de dos conjuntos de variables no fiscales (vector NF) y variables fiscales (vector VF):

$$
\operatorname{des}_{i, t}=\alpha+\beta \sum_{j=1}^{r} N F_{i, t}^{j}+\sum_{b=1}^{k-1}\left(D_{b}-D_{k}\right) V F_{i, t}^{b}+u_{i, t}
$$

Se considera que los impuestos directos son equivalentes a los impuestos distorsionadores, mientras que los impuestos indirectos son equivalentes a los impuestos no distorsionadores. Asimismo, el gasto público corriente es equivalente a los gastos improductivos, mientras que la inversión pública es equivalente a gastos productivos.

\section{Evolución y comportamiento de las variables}

De acuerdo con datos del Instituto Nacional de Estadística y Geografía (INEGI) en 2019 el producto interno bruto se contrajo 0.15 por ciento, principalmente debido a la desaceleración ocurrida en la producción industrial y los servicios, aunque en los años anteriores había una leve caída. Esto se puede observar en la gráfica 1. 


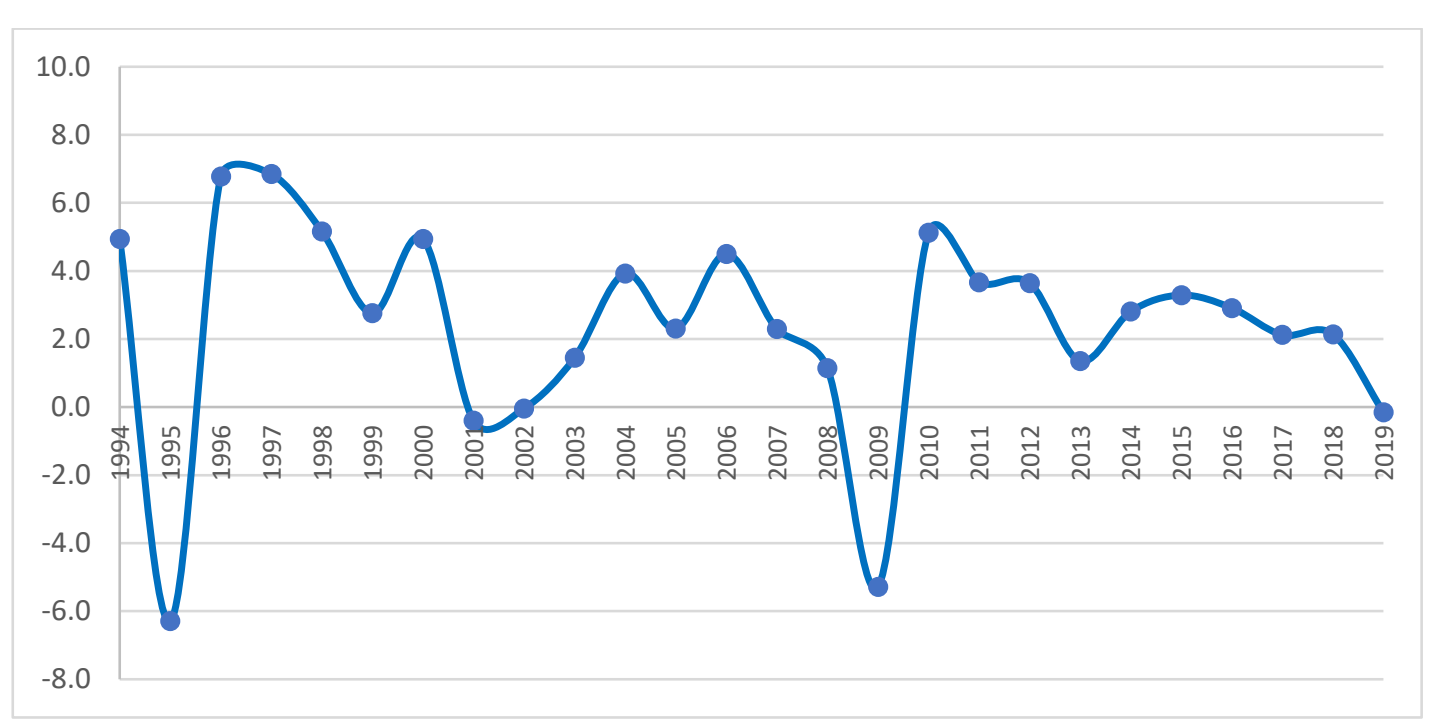

Gráfica 1. Tasa de crecimiento del Producto Interno Bruto

Fuente: elaboración propia con base en datos de INEGI.

Esta caída en la producción también puede deberse a conflictos ocurridos que afectaron la inversión, por ejemplo, ocurrió el conflicto de huelgas en las manufactureras en Tamaulipas, la escasez de gasolina, los bloqueos ferroviarios, así como los recortes en los gastos públicos implementados en el inicio de este sexenio.

La desigualdad de ingresos mide la distribución interpersonal del ingreso, capturando cómo se distribuyen los ingresos individuales o familiares entre la población en un momento determinado. La desigualdad se puede ver desde diferentes perspectivas, cada una de las cuales puede proporcionar información sobre la naturaleza, las causas y las consecuencias de la desigualdad y también puede tener diferentes implicaciones políticas. En la gráfica 2 se observa mediante el índice de Gini que en la última década la desigualdad en México ha disminuido ligeramente.

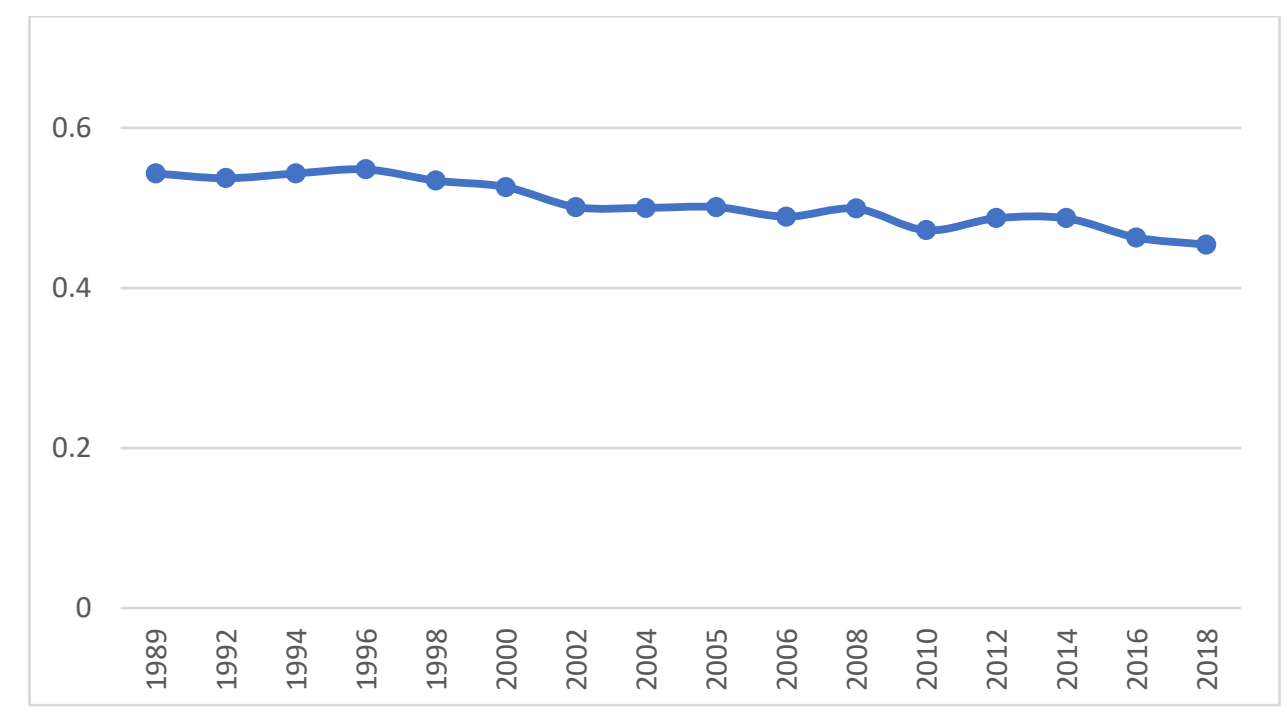

Gráfica 2. Evolución de la desigualdad en México Fuente: elaboración propia con base en datos de CONEVAL e INEGI. 
Uno de los argumentos de esa disminución es que el crecimiento promedio de los ingresos fue más fuerte para los hogares de bajos ingresos que para los de altos ingresos, lo que contribuyó a una modesta disminución de la desigualdad. Según Lustig (2018) esta caída en la desigualdad a veces se ha asociado con la implementación del Tratado de Libre Comercio de América del Norte.

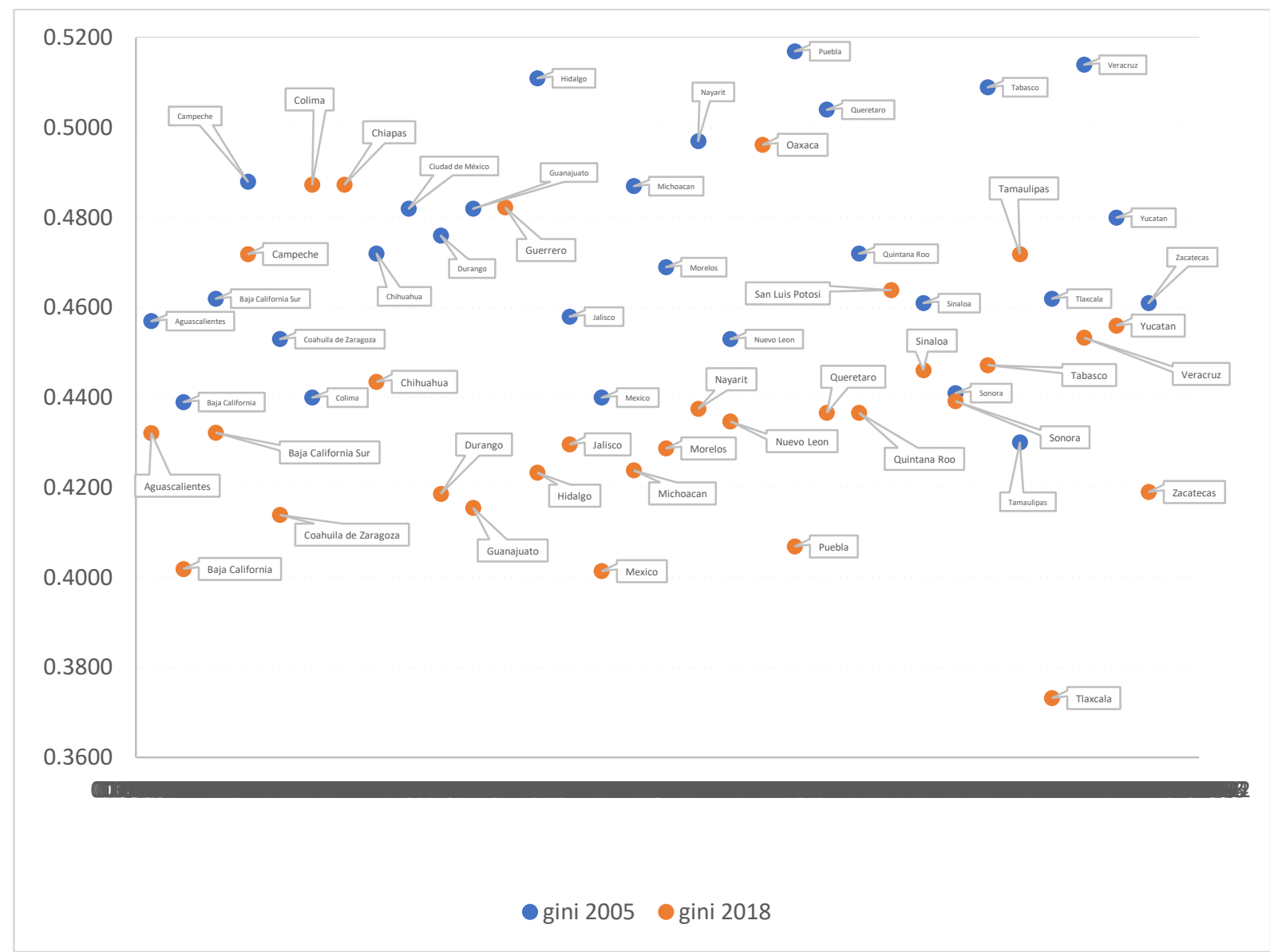

Gráfica 3. Desigualdad por entidad federativa 2005 y 2018

Fuente: elaboración propia.

De acuerdo con información ${ }^{4}$ de la Secretaria de Hacienda y Crédito Público de enero a julio de 2020 los ingresos tributarios se mantuvieron prácticamente constantes en términos reales respecto a los del mismo periodo de 2019, al presentar una disminución de 0.8 por ciento anual. De hecho, en los último cuatro años los ingresos tributarios se mantienen en alrededor del 13 por ciento como porcentaje del pib (véase grafica 4).

${ }^{4}$ Comunicado 067, 28 de agosto de 2020. 


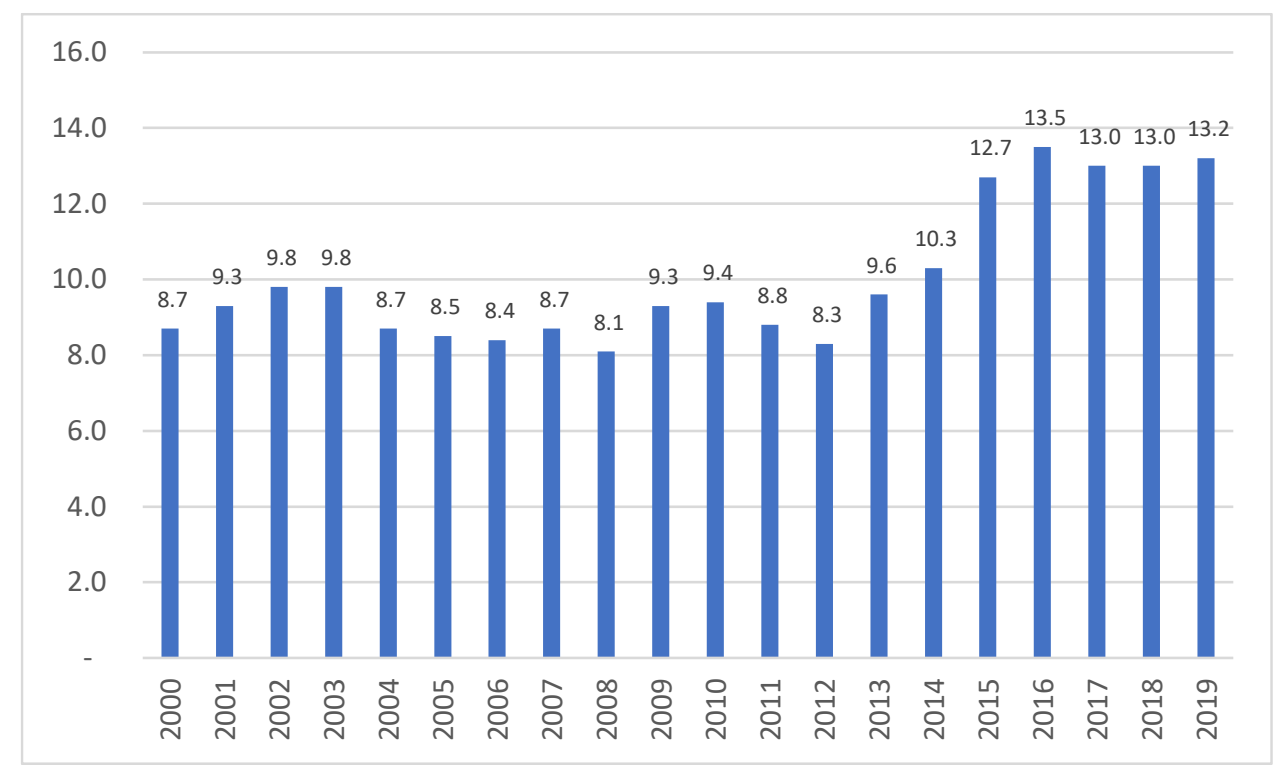

Gráfica 4. Evolución de los ingresos tributarios como porcentaje del PIB Fuente: elaboración propia.

Por su parte, de enero a julio de 2020 el gasto neto presupuestario se ubicó en 3 billones 438.6 mil millones de pesos, el cual tuvo un incremento anual de 2.4 por ciento en términos reales, el cual fue resultado del dinamismo del gasto programable que presentó un crecimiento anual de 4.8 por ciento real. Su evolución anual se puede ver la gráfica 5.

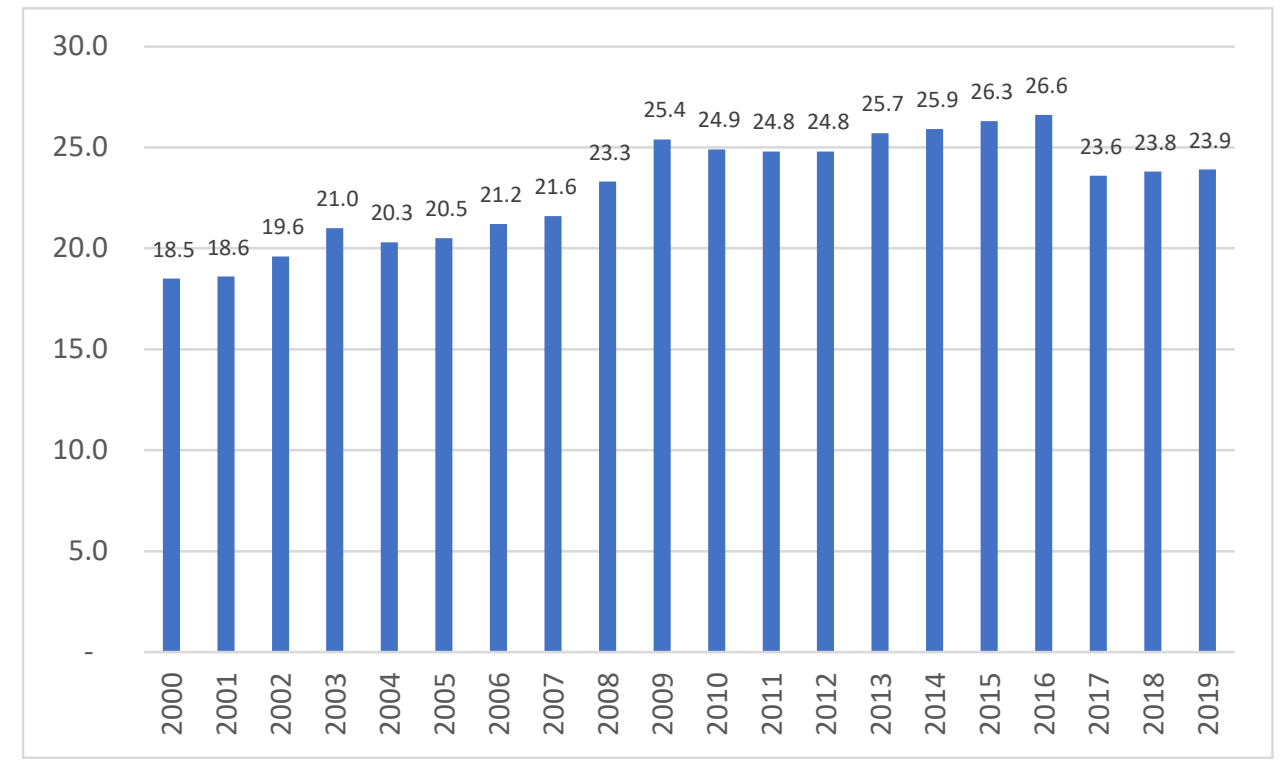

Gráfica 5. Evolución del gasto neto presupuestario

Fuente: elaboración propia. 


\section{Metodología y datos}

El modelo que se utiliza es un modelo de datos panel debido a que en este estudio se posee una estructura de información formada por muestras transversales de las entidades federativas observadas en varios años. Según Baltagi (1995) los datos del panel se refieren a conjuntos de datos que consisten en observaciones múltiples en cada unidad de muestreo. Esto puede generarse agrupando las observaciones de series de tiempo a través de una variedad de secciones transversales que incluyen países, estados, regiones, empresas o individuos u hogares muestreados al azar. Algunas de las ventajas de los modelos de datos panel son que son un conjunto de datos mucho más amplio con más variabilidad y menos colinealidad. Otra ventaja de los conjuntos de datos de panel es su capacidad para controlar la heterogeneidad individual. No controlar estos efectos específicos individuales no observados conduce a un sesgo en las estimaciones resultantes. Los conjuntos de datos del panel también son más capaces de identificar y estimar efectos que simplemente no son detectables en secciones transversales puras o datos de series de tiempo puras.

Tamara (1997) menciona que una forma de representar el modelo de datos puede ser de la manera siguiente:

$$
Y_{i t}=\alpha_{i t}+X_{i t} \beta+u_{i t}
$$

con $i=1, \ldots, N ; t=1, \ldots \mathrm{T}$, donde $i$ se refiere al individuo o a la unidad de estudio (corte transversal), $t$ a la dimensión en el tiempo, $\alpha$ es un vector de interceptos de $n$ parámetros, $\beta$ es un vector de $K$ parámetros y $X_{i t}$ es la $i$-ésima observación al momento $t$ para las $K$ variables explicativas. En este caso, la muestra total de las observaciones en el modelo vendría dado por $N$ x $T$.

De acuerdo con Greene (2002), el modelo de datos panel consta de tres modelos: el de datos agrupados, el de efectos fijos donde el efecto específico del individuo es una variable aleatoria que se puede correlacionar con las variables explicativas y el de efectos aleatorios donde el efecto específico del individuo es una variable aleatoria que no está correlacionada con las variables explicativas.

Las variables económicas, relacionadas con el producto y la inversión, se toman del Instituto Nacional de Estadísticas y Geografía. Las variables fiscales como impuestos y gasto corriente se toman de la Secretaría de Hacienda y Crédito Público. Las variables de educación se toman de la Secretaría de Educación Pública. La variable de desigualdad (Gini) se toma del Consejo Nacional de Evaluación la Política de Desarrollo Social.

Se consideran datos bianuales debido a que, aunque para la mayoría de las variables se disponen observaciones anuales, los datos sobre los coeficientes de Gini son más limitados y solo tienen datos cada dos años. 


\section{Análisis empírico}

Los modelos planteados toman como referencia las ecuaciones (6) y (7) del apartado dos y relacionan al pib per cápita y la desigualdad con variables fiscales, de inversión y producción. De la ecuación (6) se estiman 4 modelos cuyos resultados se observan en el cuadro 1.

Cuadro 1. Resultados de la estimación del modelo pib per cápita

\begin{tabular}{|c|c|c|c|c|}
\hline \multicolumn{5}{|c|}{ Variable dependiente: pib per cápita } \\
\hline Variables independientes & Modelo 1 & Modelo 2 & Modelo 3 & Modelo 4 \\
\hline \multirow[t]{2}{*}{ Impuestos directos } & -0.115538 & & -0.120441 & \\
\hline & $(0.025)$ & & $(0.021)$ & \\
\hline \multirow[t]{2}{*}{ Impuestos indirectos } & & -0.115193 & & 0.017178 \\
\hline & & $(0.173)$ & & $(0.523)$ \\
\hline \multirow[t]{2}{*}{ Gasto público corriente } & -0.284489 & -0.118398 & -0.283801 & -0.110357 \\
\hline & $(0.008)$ & $(0.069)$ & $(0.009)$ & $(0.201)$ \\
\hline \multirow[t]{2}{*}{ Inversión pública } & 0.021638 & 0.023449 & 0.0231215 & 0.0248284 \\
\hline & $(0.126)$ & $(0.394)$ & $(0.395)$ & $(0.367)$ \\
\hline \multirow[t]{2}{*}{ Crecimiento de la población } & -0.118905 & -0.108287 & -0.079261 & -0.071518 \\
\hline & $(0.392)$ & $(0.443)$ & $(0.576)$ & $(0.621)$ \\
\hline \multirow[t]{2}{*}{ Inversión privada } & 0.795598 & 0.533953 & 0.648855 & 0.347252 \\
\hline & $(0.039)$ & $(0.055)$ & $(0.045)$ & $(0.063)$ \\
\hline \multirow[t]{2}{*}{ pib } & 0.154979 & 0.361564 & 0.457458 & 0.359364 \\
\hline & $(0.008)$ & $(0.033)$ & $(0.008)$ & $(0.975)$ \\
\hline \multirow[t]{2}{*}{ educación } & 0.170482 & 0.184784 & 0.174056 & 0.189144 \\
\hline & $(0.023)$ & $(0.095)$ & $(0.014)$ & $(0.085)$ \\
\hline \multirow[t]{2}{*}{ desigualdad } & & & -0.811281 & -0.731173 \\
\hline & & & $(0.089)$ & $(0.042)$ \\
\hline \multirow[t]{2}{*}{ Constante } & 4.128218 & 0.334632 & -3.796463 & 0.133972 \\
\hline & $(0.355)$ & $(0.937)$ & $(0.395)$ & $(0.975)$ \\
\hline $\mathrm{F}(\mathrm{p}$-value $)$ & $(0.0000)$ & $(0.0000)$ & $(0.0000)$ & $(0.0000)$ \\
\hline LM(p-value) & $(0.0000)$ & $(0.0000)$ & $(0.0000)$ & $(0.0000)$ \\
\hline Hausman (p-value) & $(0.0000)$ & $(0.0000)$ & $(0.0000)$ & $(0.0000)$ \\
\hline R cuadrado & 0.1933 & 0.1725 & 0.2009 & 0.1787 \\
\hline Observaciones & 224 & 224 & 224 & 224 \\
\hline
\end{tabular}

Fuente: elaboración propia.

En el cuadro 1 se muestran los resultados de los modelos econométricos de crecimiento. En la parte superior se muestra la variable dependiente, en la primera columna se muestran las variables independientes, de las columnas dos a la cinco se muestran los modelos estimados. Las pruebas estadísticas a los modelos arrojaron que el modelo de efectos fijo fue mejor que el de datos agrupados, F(p-value) y, que el de efectos aleatorios fue mejor que datos agrupados LM(p-value). 
Asimismo, en los cuatro modelos la prueba de Hausman arrojó que el mejor modelo fue el de efectos fijos, Hausman (p-value). Se observa que los impuestos directos tienen un efecto negativo y estadísticamente significativo en el crecimiento económico. Sin embargo, los impuestos indirectos no son estadísticamente significativos, es decir, no tienen un impacto significativo en el crecimiento. Por su parte el gasto corriente es estadísticamente significativo y muestra un impacto negativo en el crecimiento, esto puede deberse a que de alguna manera el gasto no es eficiente y por el contrario puede ser malgastador, también puede deberse a que gran parte de ese gasto no se utilice con fines productivos sino con actividades de legislación o seguridad. La inversión pública arroja un coeficiente positivo, pero no es significativo, lo cual es contradictorio con lo que dicta la teoría ya que se supone que la inversión en infraestructura debería generar crecimiento económico, pero en este caso no es así. Por su parte, la inversión privada arroja un coeficiente positivo y significativo, lo cual significa la inversión privada promueve más crecimiento. Por su parte, la variable de capital humano o educación arroja un coeficiente positivo y significativo, el cual es consistente con lo que dicta la teoría del crecimiento endógeno. Respecto a la variable desigualdad medida por el coeficiente de Gini arrojó un efecto negativo y estadísticamente significativo, lo cual indica que el aumento de la desigualdad perjudica el crecimiento económico. Este resultado es coherente con lo que mencionó el fondo monetario internacional donde menciona que, si aumenta la brecha desigual entre ricos y pobres, esto provoca un reducción del producto interno bruto, aunque el signo es contradictorio con estudios hechos por Lundberg y Squire (2003) y Castelló-Climent (2010) quienes obtienen un signo distinto.

De la ecuación (7) se estiman cuatro modelos donde la variable dependiente es la desigualdad cuyos resultados se observan en el cuadro 2 .

Cuadro 2. Resultados del modelo de desigualdad

\begin{tabular}{|lcccc|}
\hline Variable dependiente: desigualdad & & & & \\
\hline \multicolumn{1}{|c}{ Variables independientes } & Modelo 1 & Modelo 2 & Modelo 3 & Modelo 4 \\
\hline Impuestos directos & -0.717691 & & -0.145234 & \\
Impuestos indirectos & $(0.037)$ & & $(0.004)$ & \\
& & 0.149887 & & 0.188746 \\
Gasto público corriente & & $(0.041)$ & & $(0.015)$ \\
& -0.019746 & -0.032467 & -0.079308 & -0.028078 \\
Inversión pública & $(0.080)$ & $(0.000)$ & $(0.351)$ & $(0.001)$ \\
& -0.043409 & -0.054417 & 0.025741 & -0.043323 \\
Inversión privada & $(0.075)$ & $(0.156)$ & $(0.049)$ & $(0.178)$ \\
& -0.182032 & -0.186792 & -0.179164 & -0.226765 \\
pib & $(0.046)$ & $(0.048)$ & $(0.093)$ & $(0.002)$ \\
& & & 0.176315 & -0.187642 \\
educación & & & $(0.027)$ & $(0.339)$ \\
& 0.210947 & 0.310379 & 0.152875 & 0.351878 \\
Constante & $(0.048)$ & $(0.481)$ & $(0.027)$ & $(0.014)$ \\
& 0.9749788 & 1.106347 & 0.3470925 & 1.488027 \\
& $(0.000)$ & $(0.000)$ & $(0.057)$ & $(0.001)$
\end{tabular}




\begin{tabular}{|lcccc|} 
& & & & \\
F(p-value) & $(0.0000)$ & $(0.0000)$ & $(0.0000)$ & $(0.0000)$ \\
LM(p-value) & $(0.0000)$ & $(0.0000)$ & $(0.0000)$ & $(0.0000)$ \\
Hausman (p-value) & $(0.0073)$ & $(0.0002)$ & $(0.1401)$ & $(0.0034)$ \\
R cuadrado & 0.1466 & 0.1412 & 0.1256 & 0.1454 \\
Observaciones & 224 & 224 & 224 & 224 \\
\hline
\end{tabular}

Fuente: elaboración propia.

En el cuadro 2 se muestran los resultados de los modelos econométricos de desigualdad. Al igual que en el cuadro anterior la interpretación es muy similar salvo que la tercera estimación el mejor modelo es el de efectos aleatorios, la prueba de Hausman arroja un valor de 0.1401. Los resultados arrojan que los impuestos directos tienen un efecto negativo en la desigualdad, esto podría explicarse en que los de mayor ingreso deberían tener una tasa impositiva más alta que los de menor ingresos, por el contrario, el coeficiente de los impuestos indirectos es positivo y significativo. Por su parte, el gasto público corriente arroja una relación negativa salvo el modelo tres, esto indica que dicho gasto contribuye a reducir la desigualdad, esto podría ser a través de los efectos redistributivos que tendría el gasto a través de los programas sociales. Por su parte, el coeficiente de la inversión pública es negativa y significativo en dos modelos, pero en otros dos no los es, aunque el impacto es pequeño dada la magnitud del coeficiente arrojado, esto indica que la inversión pública podría reducir en mayor medida la desigualdad siempre y cuando se desarrollasen mayores infraestructuras que se vinculen a núcleos de actividad económica que generen el potencial desarrollo de actividades productivas generadoras de empleo. Por su parte, la inversión privada arroja un coeficiente negativo y significativo. Por su parte, el pib tiene una relación positiva, esto indica que el poco crecimiento que puede haber no contribuye a reducir la desigualdad. Respecto a la educación el modelo arroja un coeficiente positivo y significativo, esto indica que la educación no está generando reducción de la desigualdad, este resultado contrario de acuerdo con la teoría del capital humano.

\section{Conclusiones}

El objetivo de este documento fue analizar de qué manera instrumentos de política fiscal influyen crecimiento y en la desigualdad del ingreso en México. Par lo cual se expusieron antecedentes teóricos y se utilizó un modelo de crecimiento endógeno que incorpora variables fiscales. Asimismo, se hizo uso de simulaciones econométricas para evaluar la incidencia de variables fiscales en crecimiento y desigualdad.

Respecto al modelo de crecimiento, los resultados muestran que el gasto corriente no ejerce un impacto positivo en el crecimiento, esto podría ser debido que posiblemente existe ineficiencia en la ejecución del gasto o que posiblemente se sea utilizado en actividades que no generan actividades productivas. Los impuestos directos ejercen un impacto negativo en el crecimiento, mientras que los impuestos indirectos no arrojan resultados significativos en el modelo. Por su parte, la inversión pública no está siendo significativa para contribuir al crecimiento, esto indica que no está adecuadamente dirigida a generar medios que incentiven el desarrollo de actividad económica productiva. Respecto al modelo de desigualdad, los resultados muestran que el gasto corriente 
contribuye a reducir la desigualdad, como ya se mencionó, esto podría ser a través de los efectos redistributivos que tendría el gasto a través de los programas sociales. De igual forma, los impuestos directos contribuyen a una reducción de dicha desigualdad, mientras que los impuestos indirectos contribuyen a aumentarla. Respecto a la inversión pública se encuentran resultados divididos, ya que dos modelos indican que la inversión pública contribuye a reducir la desigualdad, mientras que los otros dos arrojan resultados contrarios.

La política fiscal puede ser una herramienta potente para lograr los objetivos redistributivos de un gobierno. Afecta el bienestar del hogar a través de pagos monetarios (impuestos y transferencias) y la provisión de beneficios especiales (por ejemplo, educación gratuita y servicios de salud).

En algunos casos, las políticas redistributivas no solo ayudan a alcanzar los objetivos de equidad, sino que también mejoran la eficiencia, especialmente cuando abordan las imperfecciones del mercado. La falta de acceso al crédito por parte de los hogares pobres, por ejemplo, es una imperfección importante del mercado que impide que los hogares pobres soliciten préstamos para financiar la educación. En estas circunstancias, el financiamiento gubernamental de la educación puede ayudar a las familias a superar este obstáculo al tiempo que fortalece la equidad y la acumulación eficiente de capital humano.

Las políticas de impuestos y gastos deben diseñarse con sabiduría para minimizar cualquier efecto adverso sobre los incentivos para trabajar, ahorrar e invertir. Por el lado de los ingresos, esto implica construir bases impositivas más amplias y confiables mediante la reducción de exenciones, la lucha contra la evasión fiscal y el fortalecimiento de la administración. Por el lado del gasto, las prioridades incluyen ampliar el acceso a la educación y la salud, lo que reforzará la igualdad de oportunidades, y una mejor focalización de los beneficios sociales hacia los pobres. Por lo tanto, en la medida en que las políticas de impuestos y gasto del gobierno afecten el nivel del producto interno bruto, también tendrán implicaciones para la desigualdad.

\section{Referencias}

[1] Aghion, P. and Bolton, P. (1997). A Theory of Trickle-down Growth and Development. Review of Economic Studies, 64, 151-172.

[2] Alesina, A.F., Ardagna, S. (2009). Large Changes in Fiscal Policy: Taxes versus Spending. NBER Working Paper Series 15438.

[3] Auerbach, A.J. and Kotlikoff, L.J. (1987). Dynamic Fiscal Policy. Cambridge University Press.

[4] Barreix, A., J. Roca and L. Villela (2007). Fiscal Policy and Equity. Estimation of the Progressivity and Redistributive Capacity of Taxes and Social Public Expenditure in the Andean Countries. InterAmerican-Development-Bank. INTAL-INT Working Paper 33.

[5] Barro, R. J. (1990). Government spending in a simple model of endogenous growth. Journal of Political Economy, 98(1), 103-117.

[6] Brosio, G., Jiménez J. P. y Ruelas I. (2017). Analizando el impacto de la política fiscal sobre la desigualdad personal y regional: el caso de México. Artículo para ser presentado en las VI Jornadas Iberoamericanas de Financiación Local, Septiembre, 2017, Córdoba, Argentina. 
[7] Cass, D. (1965). Optimum Growth in an Aggregative Model of Capital Accumulation. Review of Economic Studies, 32, 233-240.

[8] Castelló-Climent, A. (2010). Inequality and Growth in Advanced Economies: An Empirical Investigation, Journal of Economic Inequality, 8, 293-321.

[9] Chamely, C. (1986). Optimal Taxation of Capital Income in General Equilibrium with Infinite Lives. Econometrica, 54(3), 607-622.

[10] Chu, Ke-young, H. Davoodi, and S. Gupta (2000). Income Distribution and Tax and Government Social Spending policies in Developing Countries. IMF Working Paper, No. 10/112, May.

[11] Chung, K. and Turnovsky, S.J. (2010). Foreign debt supply in an imperfect international capital market: Theory and evidence, Journal of International Money and Finance, 29, 201-223.

[12] Cubero, R. and I. Hollar (2010). Equity and fiscal policy: The Income Distribution Effects of Taxation and social Spending in Central America. IMF Working Paper, No. 00/62, March.

[13] Deaton. A.S. and Paxson, C.H. (1997). The Effects of Economic and Population Growth on National Saving and Inequality, Demography of Aging, 34(1), 97-114.

[14] Diamond, P. (1965). National Debt in a Neoclassical Growth Model. American Economic Review, 41, 1126-1150.

[15] Easterly, W. and S. Rebelo (1993). Fiscal policy and economic growth. Journal of Monetary Economics, 32(3), 417-458.

[16] Feldstein, M.S. (1974). Incidence of a Capital Income Tax in a Growing Economy with Variable Savings Rates, Review of Economic Studies, 41, 505-513.

[17] Galor, O. and Zang, H. (1997). Fertility, Income Distribution and Economic Growth: Theory and Crosscountry Race Obviousness, Japan and the World Economy, 9(2), 197- 229.

[18] García-Peñalosa, C. and Turnovsky, S.J. (2007). Growth, Inequality, and Fiscal Policy with Endogenous Labour Supply: What are the Relevant Tradeoffs? Journal of Money, Credit and Banking, (39), 369-394.

[19] Giorgio Brosio, Juan Pablo Jiménez e Ignacio Ruelas (2017). Analizando el impacto de la política fiscal sobre la desigualdad personal y regional: el caso de México. VI jornadas Iberoamericanas de Financiación Local, septiembre, 2017, Córdoba, Argentina.

[20] Goñi, E., H. J. Lopez, and L. Serven (2008). Fiscal Redistribution and Income Inequality in Latin America. World Bank Policy Research Working Paper 4487.

[21] Huang H-C. (R.), Lin, Y.-C. and Yeh, C.-C. (2009). Joint Determinations of Inequality and Growth, Economic Letters, 103, 163-166.

[22] Judd, K.L. (1985). Redistributive Taxation in a Simple Perfect Foresight Model. Journal of Public Economics, 8(1), 59-83.

[23] King, R. and Rebelo, S. (1990). Public Policy and Economic Growth: Developing Neoclassical Implications, Journal of Political Economy, 98(5), 126-150.

[24] Kitao, S. (2010). Short-run fiscal policy: Welfare, redistribution and aggregate effects in the short and long-run, Journal of Economic Dynamics \& Control, 34, 2109-2125.

[25] Koopmans, T.C. (1965). On the Concept of Optimal Growth. The Econometric Approach to Development Planning, Rand McNally.

[26] Kuznets, S. (1955). Economic Growth and Income Inequality. American Economic Review, XLV(1), 128.

[27] Levy Orlik, Noemí (2016). Política fiscal y desequilibrios económicos: el impacto de la composición del gasto público sobre el crecimiento de la economía mexicana, Economía UNAM, 13(39), 82-105.

[28] Li, H., L. Xu, and H. Zou (2000). Corruption, Income Distrubution, and Growth. Economics and Politics, 12, pp. 155-182.

[29] Lin S.-C., Kim, D.-H., Huang, H.-C. and Yeh, C.-C. (2009). Nonlinearity between Inequality and Growth, Studies in Nonlinear Dynamics Econometrics, 13(2), art. 3. 
[30] López, R., T. Vinod, and Y. Wang (2010). The Effect of Fiscal Policies on the Quality of Growth, IEGWorld Bank - IFC - MIGA. Chapter 4.

[31] Lucas, R. (1990). Supply-side Economics: An Analytical Review, Oxford Economic Papers, 42(2), 293316.

[32] Lustig, Nora (2018). El impacto del sistema tributario y el gasto social en la distribución del ingreso y la pobreza en América Latina. Una aplicación del marco metodológico del proyecto Compromiso con la Equidadad (CEQ). CEQ Institute. Documento de trabajo 62. Mayo 2018.

[33] Mookherjee, D. and Ray, D. (2003). Persistent Inequality. Review of Economic Studies, 70, 369-393.

[34] Muinelo-Gallo, L., Roca-Sagalés, O. (2011b). Economic Growth and Inequality: The Role of Fiscal Policies, Australian Economic Papers, 50 (2-3), 74-97.

[35] Perotti, R. (1996). Growth, Income Distribution and Democracy: What the Data Say. Journal of Economic Growth, 1(2), 149-187.

[36] Persson, T. and Tabellini, G. (1994). Is Inequality Harmful for Growth? American Economic Review, 84, 600-621.

[37] Piketty, T. (1997). The Dynamics of the Wealth Distribution and the Interest Rate with Credit Rationing. Review of Economic Studies, 64(2), 173-189.

[38] Rebelo, S. (1991). Analysis and Long-run Growth. Journal of Political Economy, 99(3), 500-521.

[39] Sato, K. (1967). Taxation and Neo-Classical Growth. Public Finance, 22( 3), 346-370.

[40] Summers, L.H. (1981). Capital Taxation and Accumulation in a Life Cycle Growth Model', American Economic Review, 71( 4), 533-544.

[41] Venegas-Martínez, F. (2010). Fiscal Policy in a Stochastic Model of Endogenous Growth: the Mexican Case. Indian Development Review, 8(1-2), 139-157.

[42] Venegas-Martínez, F. (2009). Un modelo estocástico de equilibrio macroeconómico: acumulación de capital, inflación y política fiscal. Investigación Económica, 68(268), 69-114.

[43] Venegas-Martínez, F. (2005). A Stochastic Model of Endogenous Growth: The Mexican Case 1930-2002. Análisis Económico, 20(43), 83-100.

[44] Wolf, E.N., Zacharias, A. (2007). The Distributional Consequences of Government Spending and Taxation in the U.S., 1989 and 2000, Review of Income and Wealth 53(4), 692-715 